\title{
La capacitación docente para una educación remota de emergencia por la pandemia de la COVID-19
}

\author{
María Obdulia González Fernández \\ Doctorada en Sistemas y Ambientes Educativos/Profesora de tiempo completo \\ del Departamento de Ingenierías de la Universidad de Guadalajara (México) \\ ogonzalez@cualtos.udg.mx | https://orcid.org/0000-0001-5890-7666
}

\section{Extracto}

La crisis sanitaria provocada por la pandemia de la COVID-19 ha revolucionado la forma de docencia en todos los niveles. Por tal motivo, el objetivo de este trabajo es analizar y evaluar una experiencia de capacitación docente tecnopedagógica para afrontar los retos de la educación a distancia. Para ello se utilizó un estudio de corte cuantitativo-exploratorio, de tipo longitudinal (precurso-poscurso), de alcance descriptivo, a partir de una muestra de 337 docentes de bachiIlerato, quienes participaron en un curso de capacitación bajo el modelo TPACK (technological pedagogical content knowledge). Los resultados demostraron una habilitación docente en lo tecnopedagógico respecto al diagnóstico; sin embargo, la competencia de evaluación en línea mostró menor desempeño. Se detectó una actitud proactiva para el uso de la tecnología en la docencia. Como sugerencia se propone que los programas de capacitación docente integren temáticas socioemocionales para afrontar con empatía las necesidades del estudiante, así como el uso variado de estrategias para el trabajo en línea.

Palabras clave: educación a distancia; capacitación docente; tecnología; pedagogía; actitud docente; COVID-19. 


\title{
Teacher training for an emergency remote education during the COVID-19 pandemic
}

\author{
María Obdulia González Fernández
}

\begin{abstract}
The health crisis derived from the COVID-19 pandemic has revolutionized the way of teaching at all levels. For this reason, the objective of this work is to analyze and evaluate a technopedagogical teacher training experience to face the challenges of distance education. To this end, a quantitative-exploratory study was used, longitudinal (pre-course-post-course), descriptive in scope, from a sample of 337 high school teachers, who participated in a training course under the TPACK model (technological pedagogical content knowledge). The results demonstrated a teaching qualification in the techno-pedagogical aspect regarding the diagnosis; however, online assessment competencies showed lower performance. A proactive attitude was detected for the use of technology in teaching. As a suggestion, it is proposed that teacher training programs integrate socio-emotional themes to empathize with the needs of the student, as well as the varied use of strategies for online work.
\end{abstract}

Keywords: distance education; teacher training; technology; pedagogy; teacher attitude; COVID-19.

Citation: González Fernández, M. ${ }^{a}$ O. (2021). Teacher training for an emergency remote education during the COVID-19 pandemic. Tecnología, Cienciay Educación, 19, 81-102. https://doi.org/10.51302/tce. 2021.614 


\section{Sumario}

1. Introducción

1.2. La formación continua del docente de bachillerato en México

2. Materiales y métodos

2.1. Población y muestra

2.2. Diseño de instrumentos

2.3. Procedimiento de recogida y análisis de datos

3. Resultados

3.1. Resultados cualitativos

4. Discusión

4.1. Las capacidades tecnopedagógicas del docente de educación media superior

4.2. Las necesidades de capacitación tecnopedagógica del docente

4.3. La relación que existe entre la capacitación del docente y su actitud tecnopedagógica

5. Conclusiones y futuras investigaciones

Referencias bibliográficas 


\section{Introducción}

A principios del año 2020, por recomendación de las organizaciones de salud internacionales en lo que respecta a la pandemia por la COVID-19, se produjo el cierre de las escuelas y los docentes pasaron de una docencia presencial a una enseñanza remota de emergencia en tan solo unas cuantas semanas. Para ello se implementó el uso de los medios digitales con la finalidad de fomentar la comunicación y la colaboración entre docentes y estudiantes. Es así como se inició una enseñanza online en diversos medios y de acuerdo a las posibilidades de cada uno de los centros educativos.

La educación en línea permite la flexibilidad de la enseñanza y fomenta el aprendizaje en cualquier momento y lugar, pero lo que actualmente se está viviendo no es exactamente un modelo instruccional en línea, sino acciones improvisadas. De acuerdo con Hodges et al. (2020), la enseñanza remota de emergencia considera un cambio temporal de la enseñanza, a modo alternativo, debido a las circunstancias de crisis por la COVID, en espera de que en un periodo de tiempo determinado volverá la modalidad presencial. Por esta razón, su objetivo principal es proporcionar acceso temporal a la educación, combinando cursos en línea, videoconferencias, el uso del móvil, la radio, la televisión, la tutoría presencial-virtual, entre otras soluciones, para mantener el contacto con los estudiantes.

Por su parte, Cabrales et al. (2020) sostienen que la enseñanza de emergencia a distancia es la estrategia de trasladar a un medio virtual la acción educativa, la cual, originalmente, estaba pensada para una modalidad presencial. Esto ha provocado un debate sobre la idoneidad de los docentes, el acceso a las tecnologías de la información y la comunicación (TIC) y la capacidad de las instituciones educativas para organizarse. El docente se
La enseñanza de emergencia a distancia es la estrategia de trasladar a un medio virtual la acción educativa, la cual, originalmente, estaba pensada para una modalidad presencial ve enfrentado a diversos retos, entre los que se encuentran la incertidumbre en cuanto a la regularización de las clases presenciales, la planeación adecuada de estrategias de aprendizaje y la desigualdad social de los estudiantes, por lo que virtualizar el aprendizaje es un asunto complejo y un desafío pedagógico (De Luca, 2020).

De acuerdo con Díaz Barriga (2020), falta un proceso de formación. La pandemia sorprendió a todos los niveles y los docentes no estaban preparados. Solo el $2 \%$ del profesorado está formado para trabajar digitalmente en la educación básica. De ahí la importancia de la capacitación docente en tiempos de la COVID-19. Los estudios de Dussel (2020) y 
Sánchez et al. (2020) detectan que uno de los grandes retos es la necesidad de formar al docente en aspectos como la distribución del tiempo, el conocimiento de las herramientas digitales, el diseño instruccional en las plataformas en línea, las formas de evaluación y la creación de contenidos, entre otros.

En cambio, Suárez (2020) propone que la capacitación docente debe ser continua y ha de permanecer incluso después de la pandemia, además de potenciar el autoaprendizaje y enseñar a investigar para resolver problemas del ejercicio

La capacitación docente debe ser continua y ha de permanecer incluso después de la pandemia profesional. Es importante medir el impacto de la capacitación docente en materia del uso de las TIC en los procesos de mediación y los resultados de la aplicación de dichos recursos en el aula para garantizar un mejor proceso de formación docente (Picón et al., 2020).

Una alternativa para la actualización docente en este momento de crisis es utilizar el modelo TPACK. Este modelo ha demostrado su eficacia en la capacitación docente de diferentes niveles educativos, pues parte del análisis de los conocimientos que posee el profesorado tanto en su formación como en su desempeño profesional. Este se apoya en las ideas de Shulman (1987) y fue consolidado por Mishra y Koehler (2006). Es así que integra seis conocimientos fundamentales que un docente debe desarrollar para el buen uso de las TIC en el aula: los conocimientos que giran en torno a la tecnología, el conocimiento pedagógico y tecnológico como base, así como su integración de acuerdo con el contenido disciplinar. Así se configura un conjunto de aspectos abordados en el modelo TPACK (véase figura 1).

Figura 1. Modelo TPACK

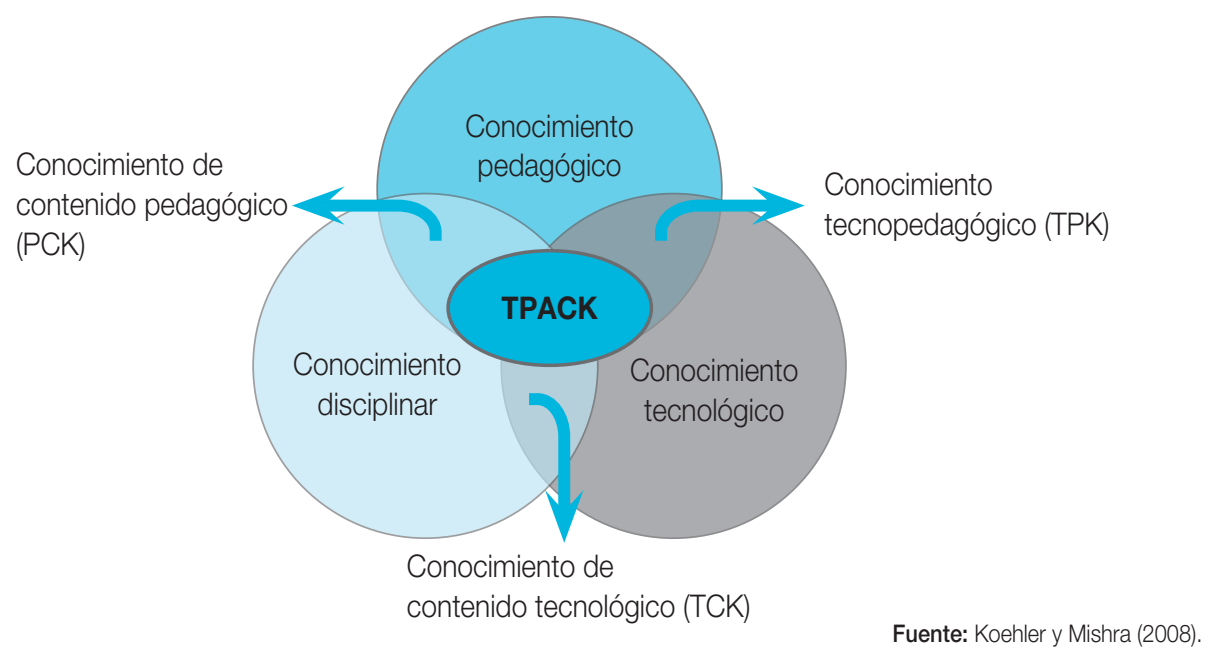


En este sentido, la formación del docente de bachillerato debe ser dialéctica, contextualizada a los cambios socioculturales para un aprendizaje integral. Por tal motivo, este modelo «ha generado expectativas en el área de la formación docente, debido a la integración de los conocimientos (tecnológico, pedagógico y contenido); además de los conocimientos que se crean con la integración de estos» (Morán et al., 2017, p. 54).

En este sentido, Cabero et al. (2015) crearon un instrumento para diagnosticar la formación del profesorado en TIC bajo el modelo TPACK con la finalidad de realizar planes de formación y capacitación docente contextual.

Ante la realidad actual de una enseñanza remota de emergencia, el docente requiere convertirse en tutor en línea, por lo que el modelo TPACK puede ser una alternativa para su habilitación, puesto que identifica y reconoce los saberes necesarios para la integración de la tecnología en la docencia (Lima y Flores, 2018), así como comprende las necesidades explícitas de formación, en cuanto a conocimientos tecnológicos, pedagógicos y disciplinares.

Según Mohamad et al. (2020), el modelo TPACK sirve como lente para evaluar las experiencias de enseñanza en línea por parte del profesorado. Y para Cardona-Londoño et al. (2020), los docentes requieren formación integral, por lo que el modelo TPACK puede ser una alternativa para guiar la capacitación.

Como consecuencia de la pandemia provocada por la COVID-19, muchas universidades realizaron campañas de preparación docente en formato de webinars, es decir, seminarios educativos y prácticos que se caracterizan por ser masivos y abiertos, y cuyas grabaciones pueden ser consultadas posteriormente.

Entre una de las experiencias previas de capacitación virtual masiva se encuentra la documentada por Guayara et al. (2019), quienes realizaron una capacitación virtual a docentes de la Amazonia. En el caso de este estudio, obtuvieron resultados positivos, entre los que se destaca el desarrollo de competencias digitales y su aceptación por parte de los docentes. Peña et al. (2012) mencionan que los cursos en entornos virtuales de aprendizaje son adecuados para la formación del docente, ya que pueden ayudar a eliminar las barreras espaciales y temporales. Asimismo, la experiencia vivencial puede ayudar en el desarrollo del uso de nuevas competencias pedagógicas, comunicativas y tecnológicas para aplicarlas al quehacer cotidiano de las aulas.

\subsection{La formación continua del docente de bachillerato en México}

La educación media superior en México, también denominado «bachillerato» o «preparatoria», se imparte en tres modelos educativos, como son el bachillerato General, el bachillerato Tecnológico y el Profesional Técnico. Dentro de estos modelos educativos operan 200 planes y programas, divididos en diferentes modalidades, como es la modalidad escolarizada, la mixta y a distancia, de acuerdo al Instituto Nacional para la Evaluación de la Educación (INEE, 2018). Ante este contexto, la actualización y formación continua de los 
docentes es compleja y variada, ya que existen diversas opciones para habilitar al personal académico, como certificaciones, cursos, talleres, congresos, entre otros.

El perfil inicial del profesorado de bachillerato es diverso, de acuerdo a las diferentes disciplinas, sin embargo, esto no quiere decir que los docentes cuenten con una formación específica para la enseñanza. En este sentido carecen de las herramientas y habilidades didácticas necesarias para la práctica docente (Lozano, 2016).

A partir de la reforma de la educación media superior en el 2008, la Secretaría de Educación Pública estableció el Acuerdo 447, que describe el conjunto de competencias docentes de bachillerato. A partir de entonces se puso en marcha el Programa de Formación Docente en Educación Media Superior (PROFORDEMS), el cual consta de tres módulos principales orientados a una formación por competencias. Otra de las acciones de formación continua a nivel gubernamental fue la Certificación de Competencias Docentes para la Educación Media Superior (CERTIDEMS). Estos dos programas constituyen acciones importantes en la conformación de un sistema nacional de formación continua en el nivel educativo.

No obstante, el informe del INEE, presentado por Medrano et al. (2015), califica la formación continua de los docentes de bachillerato como incipiente, considerando que, en este nivel educativo, los procesos de capacitación se han enfocado a la formación pedagógica del docente novel. Asimismo, Lozano (2016) menciona que la capacitación que se ofrece es con una lógica instrumental que limita el quehacer docente a un simple manejo de estrategias, de tecnologías bajo la idea de innovación.

Con la llegada de la pandemia se han puesto de manifiesto las carencias de capacitación docente en el uso pedagógico de las TIC y en el manejo de la educación a distancia, puesto que se percibía que el profesorado estaba poco preparado y que necesitaba mucho apoyo. De acuerdo con los resultados del informe de la United Nations Educational Scientific and Cultural Organization-United Nations Children's Fund (UNESCO-UNICEF, 2020), en América Latina y el Caribe, solo el $75 \%$ de los docentes han sido formados para utilizar plataformas en línea. Estos resultados son más bajos que los obtenidos en Europa y Asia.

Flores y Navarrete (2020) sostienen que al inicio de la pandemia existía una carencia de capacitación en materia virtual, por lo que el personal académico tuvo que adaptarse sobre la marcha para adquirir las habilidades necesarias mediante procesos autodidactas. Aunque en la actualidad existen gran diversidad de materiales en la web, también es cierto que los docentes requieren habilidades para la selección del material de acuerdo con sus necesidades.

Delgado (2020) menciona que, frente al reto de la educación a distancia durante la pandemia, el docente requiere tiempo para aprender y no solo enseñar. Esto es evidente para aquellos docentes con menores habilidades digitales y que buscan diferentes estrategias y alternativas para llegar a los estudiantes con desigualdades tecnológicas.

Por tal motivo, el presente trabajo tiene la intención de presentar los resultados del análisis de una experiencia de capacitación docente virtual masiva a inicios de la pandemia, 
durante los meses de abril-mayo de 2020. Cabe mencionar que los resultados de este estudio dan pautas para mejorar los procesos de capacitación docente y orientar las acciones de apoyo a la formación docente de bachillerato.

\section{Materiales y métodos}

La investigación fue de corte cuantitativo-exploratorio, con un diseño no experimental de tipo longitudinal (precurso-poscurso) y de alcance descriptivo. El principal objetivo del trabajo fue analizar los avances en la capacitación docente y su impacto en la actitud del profesorado a partir de un proceso de formación para una docencia remota de emergencia.

\subsection{Población y muestra}

La población se conformó por docentes de bachillerato Tecnológico del estado de Jalisco (México). La muestra, de tipo no probabilístico incidental, se conformó por 337 docentes voluntarios, de un total de 508 asistentes al curso de capacitación «Uso innovador de las TIC en pro del aprendizaje» celebrado en los meses de abril y mayo del 2020. Del total de la muestra, 158 fueron hombres (47\%) y 179 mujeres (53\%). Los rangos de edad de los participantes oscilaron entre menores de 30 años, el $8 \%$; entre 31 y 40 años, el $37 \%$; entre 50 y 60 años, el $15 \%$; y mayores de 60 años, el $4 \%$.

\subsection{Diseño de instrumentos}

Se realizaron dos instrumentos. El primer instrumento de autoevaluación diagnóstica constó de 18 ítems, de los cuales, los 5 primeros fueron datos generales. Otros 5 ítems tenían como propósito autoevaluar el conocimiento tecnológico de la herramienta Google Drive y de la plataforma Google Classroom, la creación de cursos en línea, la evaluación en línea y la creación de recursos digitales. Los últimos 8 ítems pretendían evaluar la actitud de los docentes respecto a la integración de las TIC en la docencia.

La validación del instrumento fue realizada por cinco expertos que revisaron el contenido. Además, se aplicó la prueba alfa de Cronbach para validar el nivel de consistencia interna, cuyo resultado obtuvo un valor de 0,913 , lo que indica que el cuestionario tenía consistencia alta.

El segundo instrumento fue de tipo mixto y constó de 30 ítems cerrados distribuidos en las siguientes categorías: datos generales (5 ítems), avance en conocimientos tecnopedagógicos ([CTP] 7 ítems), necesidades de capacitación tecnopedagógica y de contenido ([CTPC] 10 ítems) y actitud ante lo tecnopedagógico ([ATP] 8 ítems). El diseño de las preguntas corresponde a una escala Likert de 4 puntos: 0 = Nulo; 1 = Bajo; 2 = Bueno; 3 = Muy bueno; y 4 = Excelente. Para el apartado de actitud se tomó la siguiente 
escala: 5 = Muy de acuerdo; 4 = De acuerdo; 3 = Ni de acuerdo, ni en desacuerdo; 2 = En desacuerdo; y 1 = Muy en desacuerdo.

Por último, se agregaron dos preguntas abiertas respecto a la experiencia de la capacitación docente recibida y sugerencias para la docencia remota de emergencia. Para la eficacia del constructo del instrumento se aplicó el análisis factorial mediante la prueba de Kaiser-Meyer-Olkin (KMO) en cada una de las tres categorías del cuestionario.

Los resultados de la prueba KMO (véase cuadro 1) demuestran valores superiores a 0,7 y los valores de significación son menores a 0,05 , por lo que se puede confirmar que los ítems cuentan con relación en cada una de sus dimensiones. Los resultados de fiabilidad del alfa de Cronbach fueron de 0,825 , por lo que se puede decir que es un instrumento confiable.

Cuadro 1. Prueba KMO y Bartlett procesada en SPSS 22

\begin{tabular}{|c|c|c|c|}
\hline Categoría de ítem & KMO & Sig. & Bartlett (gl) \\
\hline CTP & 0,901 & 0,0 & 21 \\
\hline CTPC & 0,928 & 0,0 & 28 \\
\hline ATP & 0,793 & 0,0 & 28 \\
\hline
\end{tabular}

Nota: Sig. = Significancia; $g$ l = Grados de libertad

Fuente: elaboración propia.

\subsection{Procedimiento de recogida y análisis de datos}

Se aplicaron dos cuestionarios. El primero, de autoevaluación diagnóstica, al inicio de la capacitación docente, en formato electrónico, mediante la herramienta Google Forms, a través de un enlace que se distribuyó en la plataforma de Google Classroom, que permaneció abierto durante la primera semana del curso, en abril de 2020. El segundo instrumento se aplicó al término del curso, mediante la herramienta Google Forms, en mayo de 2020. Los datos previamente fueron procesados en una hoja de cálculo para su organización. Posteriormente, se analizaron con el programa SPSS, versión 22, aplicando diferentes análisis descriptivos de frecuencia y de medidas centrales; esto, para el caso de los dos cuestionarios.

Para las dos preguntas de carácter cualitativo se utilizó el análisis de contenido de las preguntas, por lo que se codificaron a partir de los siguientes momentos:

- Determinar el objeto de análisis (sugerencias para el curso de capacitación y recomendaciones para la educación remota de emergencia). 
- Determinación de las reglas de codificación (ítems 30 y 31).

- Elaborar un sistema de categorías de tipo inductivo.

- La codificación, que es la asignación de códigos a cada categoría, clasificando el material para la construcción de un sistema de códigos.

- Generar las inferencias a partir de un inventario en el que se aíslan las unidades de significado, conformando el contenido empírico de las categorías.

Para dicho análisis se utilizó el programa MAXQDA (software para el análisis de datos cualitativos), versión 2020, en el cual se generó el listado de códigos y segmentos. A partir de las diferentes codificaciones, se utilizó la herramienta visual de «Modelo jerárquico de códigos y subcódigos» para representar el análisis de los datos cualitativos.

\section{Resultados}

El cuestionario fue respondido por 337 docentes, de los cuales el $49 \%$ cuentan con licenciatura; el $39 \%$, con maestría; $5 \%$, con especialidad y maestría; y el 2\% restante han cursado una carrera técnica. Al principio se les aplicó una encuesta diagnóstica para conocer su percepción respecto a sus conocimientos de las herramientas tecnopedagógicas de la plataforma G Suite, así como de conceptos relacionados con la educación en línea. La comparativa entre la encuesta diagnóstica y el cuestionario de cierre demuestra que el avance más significativo estuvo relacionado con la evaluación digital, ya que se notó un incremento de 1,32 puntos promedio (véase figura 2).

Figura 2. Comparativa entre la encuesta diagnóstica y el cuestionario de cierre

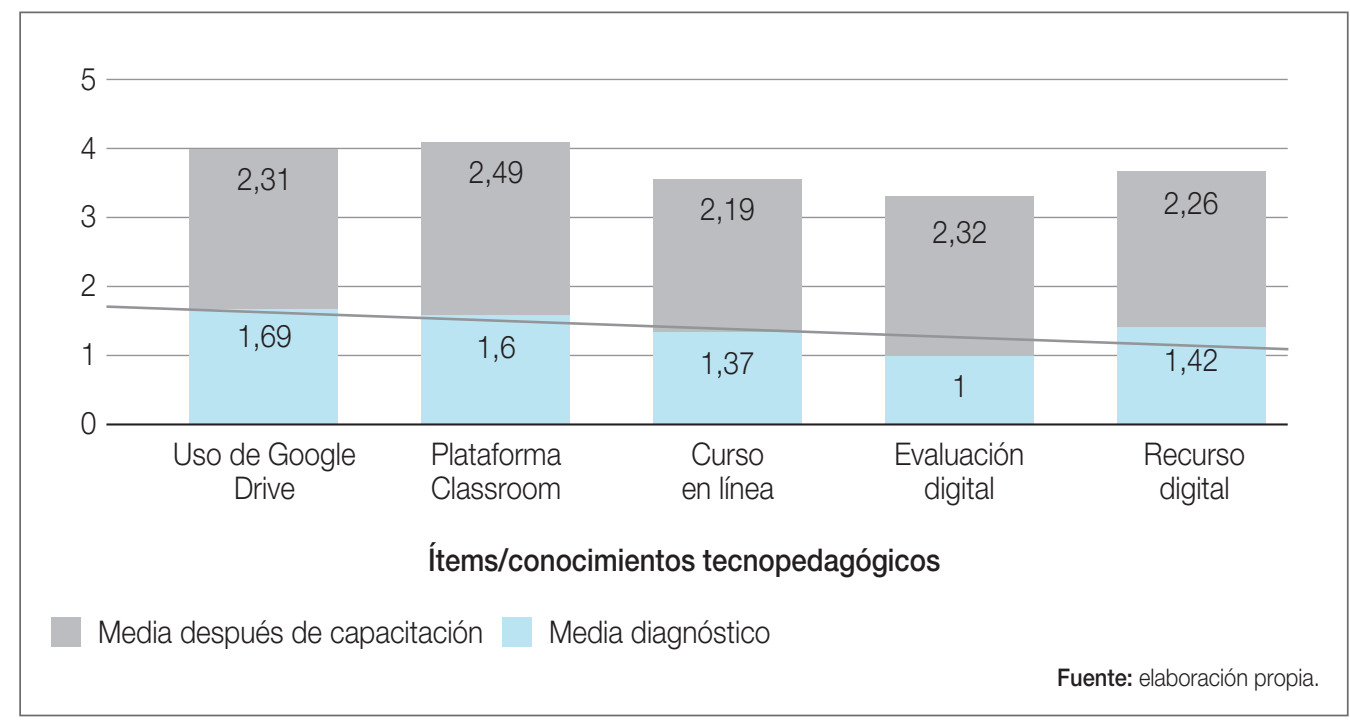


Los resultados respecto a la evaluación del avance en el conocimiento de las herramientas tecnológicas indican que la media más alta se encuentra en el uso de la herramienta Google Classroom, con un valor de 2,49 (esto se puede traducir a la escala de «Bueno» a «Muy bueno»), seguido por las herramientas de creación de cursos y el uso de Google Drive (véase cuadro 2).

Cuadro 2. Percepción del avance en los conocimientos tecnopedagógicos

\begin{tabular}{|c|c|c|c|c|c|c|c|}
\hline \multirow{2}{*}{ Ítem } & \multicolumn{5}{|c|}{ Frecuencia por escala } & \multirow{2}{*}{ Media } & \multirow{2}{*}{ Desv. 0} \\
\hline & 0 & 1 & 2 & 3 & 4 & & \\
\hline Google Drive & & 42 & 178 & 84 & 30 & 2,31 & 0,803 \\
\hline Google Classroom & & 21 & 165 & 113 & 36 & 2,49 & 0,769 \\
\hline Evaluación en línea & & 63 & 169 & 78 & 24 & 2,19 & 0,822 \\
\hline Creación de cursos & 1 & 41 & 166 & 103 & 24 & 2,32 & 0,791 \\
\hline Creación de recursos & 1 & 52 & 165 & 90 & 26 & 2,26 & 0,825 \\
\hline
\end{tabular}

Nota: 0 = Nulo; 1 = Bajo; 2 = Bueno; 3 = Muy bueno; 4 = Excelente; Desv. $\sigma=$ Desviación estándar

Fuente: elaboración propia.

Con relación a la pregunta sobre la importancia de la capacitación docente respecto a algunas temáticas, la respuesta que obtuvo mayor trascendencia fue la del desarrollo de "Cursos en línea», con una media de 3,35, seguido de la «Evaluación en línea», con 3,34 y el «Uso del móvil en el aula», con 3,30. Estos resultados se pueden observar en el cuadro 3.

Cuadro 3. Valoración de necesidades de capacitación

\begin{tabular}{|c|c|c|c|c|c|c|}
\hline \multirow{2}{*}{ Ítem } & \multicolumn{4}{|c|}{ Frecuencia por escala } & \multirow{2}{*}{ Media } & \multirow{2}{*}{ Desv. 0} \\
\hline & 1 & 2 & 3 & 4 & & \\
\hline Gamificación & 5 & 32 & 205 & 95 & 3,16 & 0,642 \\
\hline Uso del móvil en el aula & 4 & 22 & 180 & 131 & 3,30 & 0,643 \\
\hline Cursos en línea & 4 & 11 & 185 & 137 & 3,35 & 0,604 \\
\hline Diseño instruccional & 1 & 29 & 191 & 116 & 3,25 & 0,616 \\
\hline Diseño instruccional mixto & 5 & 36 & 192 & 104 & 3,17 & 0,668 \\
\hline
\end{tabular}




\begin{tabular}{|c|c|c|c|c|c|c|}
\hline \multirow{2}{*}{ Ítem } & \multicolumn{4}{|c|}{ Frecuencia por escala } & \multirow{2}{*}{ Media } & \multirow{2}{*}{ Desv. $\sigma$} \\
\hline & 1 & 2 & 3 & 4 & & \\
\hline \multicolumn{7}{|l|}{4} \\
\hline Aula invertida & 5 & 28 & 190 & 114 & 3,23 & 0,657 \\
\hline Herramientas colaborativas & 4 & 23 & 192 & 118 & 3,26 & 0,633 \\
\hline Organizadores gráficos & 4 & 37 & 195 & 101 & 3,17 & 0,656 \\
\hline Selección de información & 6 & 45 & 180 & 106 & 3,15 & 0,707 \\
\hline Evaluación en línea & 3 & 21 & 171 & 142 & 3,34 & 0,636 \\
\hline
\end{tabular}

Nota: 1 = Poco necesario; 2 = No tan necesario; 3 = Necesario; 4 = Muy necesario; Desv. $\sigma=$ Desviación estándar.

Fuente: elaboración propia.

Los resultados sobre la actitud en relación con la integración de la tecnología en los procesos educativos demuestran que la media más alta se encuentra vinculada a que «Las TIC permiten mayor flexibilidad en el aprendizaje», con una media de 4,43. Esto significa que la valoración se encuentra entre «muy de acuerdo» y «de acuerdo». Además, consideran que «Las TIC son primordiales en clase», con una media de 4,42, y que «Las TIC fomentan la creatividad», con una media de 4,32. Cabe resaltar que los ítems con valores bajos son los que denotan una actitud negativa hacia la tecnología, por lo que los docentes se encuentran en desacuerdo con ellos, como, por ejemplo, «Con las TIC se invierte más tiempo» en la práctica docente, con un valor de media de 2,27. Estos resultados se pueden observar en el cuadro 4.

Cuadro 4. Actitud frente a la tecnología (ATP)

\begin{tabular}{|c|c|c|c|c|c|c|c|}
\hline \multirow{2}{*}{ Ítem } & \multicolumn{5}{|c|}{ Frecuencia por escala } & \multirow{2}{*}{ Media } & \multirow{2}{*}{ Desv. $\sigma$} \\
\hline & 1 & 2 & 3 & 4 & 5 & & \\
\hline Las TIC son primordiales en clase & 1 & 1 & 13 & 161 & 161 & 4,42 & 0,613 \\
\hline $\begin{array}{l}\text { Las TIC permiten la flexibilidad en } \\
\text { el aprendizaje }\end{array}$ & 1 & 5 & 11 & 152 & 168 & 4,43 & 0,660 \\
\hline Las TIC son una distracción & 83 & 111 & 51 & 55 & 37 & 2,56 & 1,315 \\
\hline Las TIC fomentan la creatividad & 2 & 5 & 22 & 163 & 145 & 4,32 & 0,713 \\
\hline
\end{tabular}


Ítem

\section{Frecuencia por escala}

\section{1}

El teléfono es una distracción

$79 \quad 106$

\section{3}

5

5

58

31

2,57

1,271

Las TIC mejoran la calidad

Con las TIC se invierte más tiempo

109

120

18

172

139

4,30

0,718

Las TIC son un recurso

1120

36

51

21

2,27

1,235

Nota: 1 = Muy en desacuerdo; 2 = En desacuerdo; 3 =

Fuente: elaboración propia.

Al realizar la comparativa entre la actitud previa a la capacitación y la actitud poscapacitación, se puede ver que el avance más bajo se produjo en TIC y flexibilidad, con 0,18 puntos de diferencia, mientras que se pudo observar un mayor incremento de actitud positiva con relación a las TIC como recursos didácticos, con 0,50 puntos de aumento en la media general. Estos valores se pueden observar en la figura 3.

Figura 3. Comparativa de la actitud entre el diagnóstico y el poscuestionario

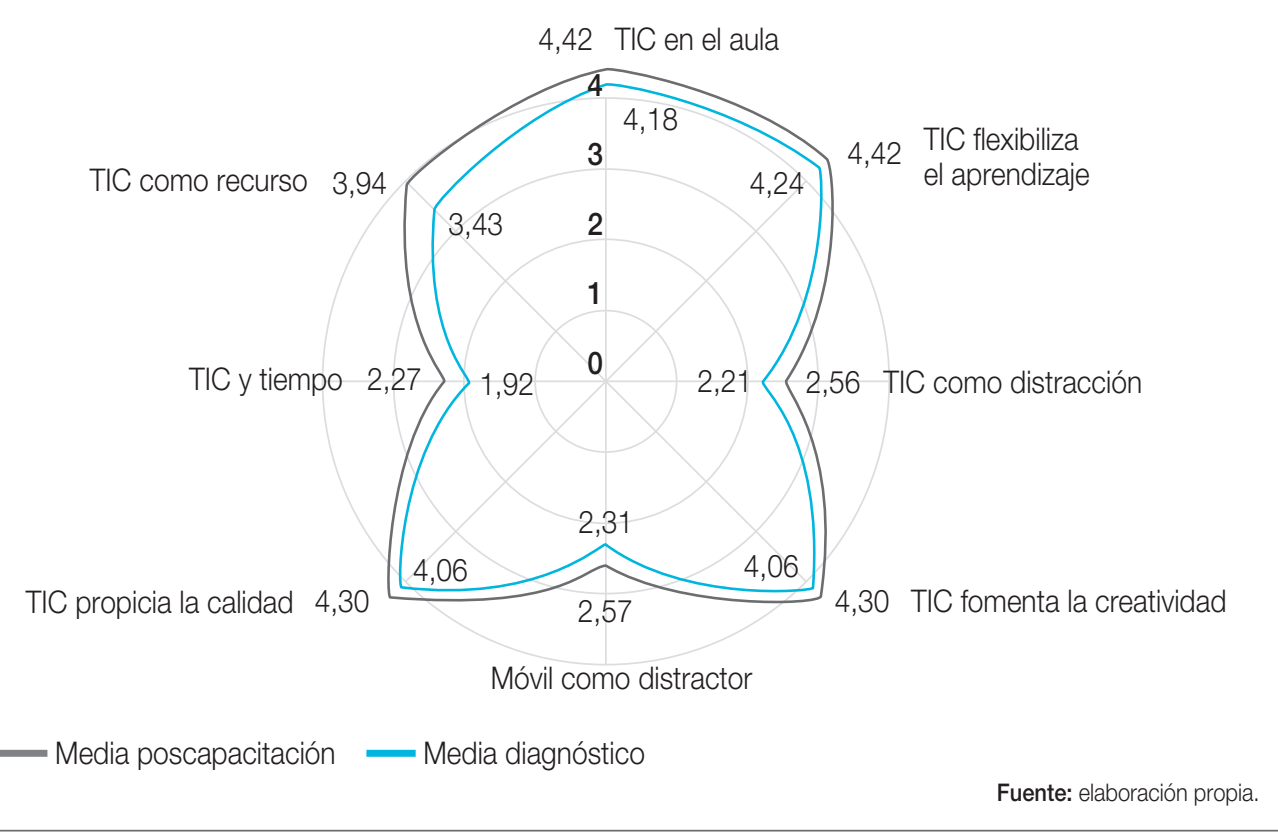




\subsection{Resultados cualitativos}

Los resultados de la parte cualitativa se dividieron en dos aspectos:

- Recomendaciones para mejorar el curso de capacitación.

- Sugerencias para una docencia remota por los efectos de la pandemia provocada por la COVID-19.

Respecto al primer aspecto, recomendaciones para mejorar el curso de capacitación, dichas sugerencias se dividieron en seis códigos: satisfacción frente a la capacitación, recomendaciones, mayor práctica, mayor tiempo, problemas de conectividad y ubicación de sesiones por nivel de competencia, y, por último, un código referido al desacuerdo con la capacitación masiva. Entre los resultados que obtuvieron mayor número de codificaciones (221) se encuentra el código de satisfacción frente al curso de capacitación, como demuestran las siguientes conclusiones de los docentes:

- «Muy enriquecedor. Aportó herramientas para mi educación continua» (E18, 4 de mayo de 2020).

- «La capacitación es muy buena. Ya manejo varias apps que nos dieron a conocer. Me fue muy útil por algunos tips que nos aportó» (E29, 4 de mayo de 2020).

Respecto al segundo aspecto, el referente a las sugerencias para una docencia remota por los efectos de la pandemia provocada por la COVID-19, fueron varios los códigos que dieron como resultado que se categorizaran en cuatro grandes rubros: actitudes docentes, pedagógico, herramientas tecnológicas y contenido tecnopedagógico. En la figura 4 se muestra un mapa del sistema de códigos y las frecuencias más altas para este aspecto.

Los docentes recomiendan tener una actitud proactiva ante las TIC. Muestra de ello es el siguiente fragmento: «Creo que lo más adecuado es estar abiertos a trabajar con nuevas tecnologías y ver de una manera positiva esta situación. Sin duda nos hace falta más capacitación o empezar a poner en práctica las nuevas tecnologías» (E93, 4 de abril de 2020).

Otra recomendación importante es mantener una actualización constante, como sostiene algún docente: «[...] actualizarse, ya que somos de diferentes áreas profesionales. A unos nos facilita y a otros, esto del uso a distancia, no complica» (E62, 4 de abril de 2020).

Los docentes recomiendan tener
una actitud proactiva ante las TIC.
Por ejemplo: «Creo que lo más
adecuado es estar abiertos a trabajar
con nuevas tecnologías y ver de
una manera positiva esta situación.
Sin duda nos hace falta más
capacitación o empezar a poner en
práctica las nuevas tecnologías»

Los docentes recomiendan tener una actitud proactiva ante las TIC. Por ejemplo: «Creo que lo más adecuado es estar abiertos a trabajar con nuevas tecnologías y ver de una manera positiva esta situación. Sin duda nos hace falta más capacitación o empezar a poner
práctica las nuevas tecnologías» 
Del mismo modo, se recomienda empatía y flexibilidad ante las circunstancias, así como adaptarse a las nuevas formas de enseñar y, por último, una actitud favorable, ya que es necesario doblar esfuerzos en esta nueva modalidad de enseñanza.

Figura 4. Modelo jerárquico de códigos sobre las recomendaciones para la educación de emergencia

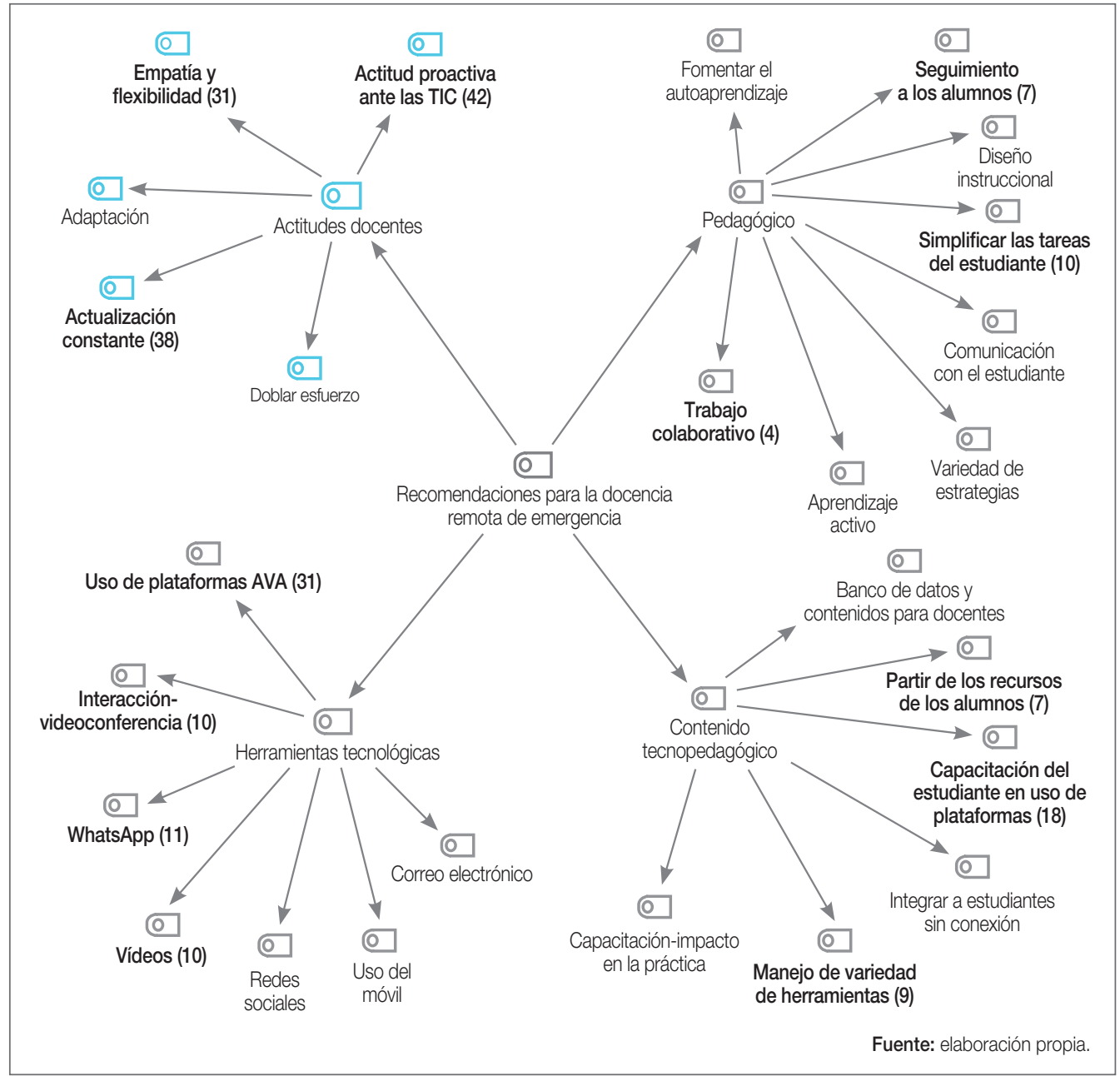

Respecto a las herramientas tecnológicas más recomendadas se encuentra el uso de plataformas educativas o ambientes virtuales de aprendizaje, como es el caso de Google Classroom, que fue una de las más frecuentes. Otra de las acciones es el uso de WhatsApp, como forma sencilla y fácil de comunicarse con los estudiantes, y el uso de sistemas de videoconferencia, como Zoom y Meet. 
En la categoría de los conocimientos pedagógicos se recomienda la simplificación de las tareas más relevantes para los estudiantes, así como el seguimiento y la retroalimentación con los mismos, el trabajo colaborativo, la comunicación constante con el alumnado y fomentar el aprendizaje activo.

Por último, en la categoría de contenido tecnopedagógico se encuentra la necesidad de capacitar a los estudiantes en las plataformas y herramientas educativas, así como que el docente parta de los recursos con los que cuenta el estudiante y maneje una variedad de herramientas para interactuar con ellos.

\section{Discusión}

\subsection{Las capacidades tecnopedagógicas del docente de edu- cación media superior}

La actual modalidad de educación remota de emergencia derivada de la pandemia provocada por la COVID-19 ha desafiado los sistemas educativos mundiales y ha dejado entrever que no todas las instituciones estaban preparadas para esta nueva realidad. Los resultados del cues-

Las habilidades con las que contaban los docentes antes de la pandemia eran insuficientes para afrontar los retos de una educación en un ambiente a distancia tionario diagnóstico precurso demuestran una carencia de los docentes en el uso y manejo de cursos para una educación a distancia. Principalmente, en el diseño instruccional y en el manejo de estrategias para la evaluación en línea es donde los resultados diagnósticos demuestran un menor conocimiento.

Esto demuestra la necesidad de capacitación docente para transitar de una educación tradicional a una educación mediada por tecnología y a distancia (Díaz Hoyos et al., 2020; Flores y Navarrete, 2020; Picón et al., 2020). Es decir, las habilidades con las que contaban los docentes antes de la pandemia eran insuficientes para afrontar los retos de una educación en un ambiente a distancia, por lo que el hecho de ser un excelente docente en un entorno presencial no garantiza el éxito cuando se actúa en entornos virtuales (Gros y Silva, 2005).

Los resultados poscurso del cuadro y de la figura 2 demuestran un avance en el desarrollo de habilidades en el manejo tecnopedagógico de herramientas como Google Drive y Google Classroom, así como en el diseño de cursos en línea y en la creación de material. Sin embargo, considerando la media de los resultados, se muestran carencias en la formación de capacidades para la evaluación formativa. 
Estos resultados concuerdan con los presentados por Fardoun et al. (2020), quienes encontraron dificultades y desconocimiento en los mecanismos para la evaluación en línea y para el seguimiento de las actividades, por lo que se concluye que se deben repensar y rediseñar las estrategias evaluativas de los procesos de enseñanza en una educación remota con una visión holística como una oportunidad para promover aprendizajes (Gallardo, 2020).

\subsection{Las necesidades de capacitación tecnopedagógica del docente}

A partir de los resultados del cuestionario poscierre se detectan las necesidades de capacitación, como la evaluación en línea, lo que demuestra una relación con los resultados de la autoevaluación de capacidades incluidos en el cuadro 2. Esto explica que, dada las características de la docencia a distancia, la evaluación del aprendizaje ha resultado un reto, principalmente en el caso de aquellos docentes para los que el examen de conocimientos es uno de los instrumentos más importantes. Por esta razón, se surgiere transitar a estrategias más inductivas, como proyectos, estudios de casos, aprendizaje basado en problemas, entre otros. Aunque los docentes han realizado un curso de capacitación, los resultados demuestran que continúan con necesidades de capacitación, como un mayor conocimiento sobre el diseño instruccional de cursos en línea, el uso del móvil en el aula y las herramientas colaborativas, como muestran los resultados del cuadro 3. El docente reconoce que necesita formarse para adaptarse a las circunstancias que se están viviendo.

De acuerdo con Gros y Silva (2005), en la enseñanza en línea, el papel del docente cambia desde una concepción tradicional a una en la que crea y dirige ambientes de aprendizaje complejos, en los que induce a los estudiantes a apropiarse del conocimiento de forma constructiva y acompaña a sus alumnos en todo momento en este proceso. Es decir, se contempla la formación del conocimiento didáctico-tecnológico. Como mencionan Díaz Hoyos et al. (2020) y Morán et al. (2017), en ocasiones no solo se requiere conocer la parte instrumental de la herramienta, sino también hay que conocer la herramienta y la didáctica propia de una asignatura frente a la virtualidad, como ocurre en el caso de las áreas de matemáticas, química, física, entre otras.

Los resultados cualitativos ofrecen una visión más concreta de lo que ha ocurrido con la experiencia de capacitación y las prácticas docentes del bachillerato en México. La experiencia de 
capacitación masiva describió la necesidad de extender la capacitación sistemática y continúa, de acuerdo con Sánchez et al. (2020). La formación docente en este periodo de emergencia debería contribuir a replantear las formas de trabajo en el aula y así desplegar propuestas para la organización de espacios, materiales y medios en que se apoya la docencia.
Uno de los retos detectados es la necesidad de consolidar las estrategias de formación y profesionalización docente en competencias tecnopedagógicas

Uno de los retos detectados es la necesidad de consolidar las estrategias de formación y profesionalización docente en competencias tecnopedagógicas. Son interesantes las sugerencias de los docentes respecto a la capacitación recibida. Un ejemplo de ello es aumentar la práctica en este tipo de cursos, dedicar mayor tiempo a la capacitación y considerar la distribución de grupos por nivel de desempeño de los participantes. En relación con los aspectos de la capacitación, el modelo TPACK ayudó a categorizar los códigos de las preguntas abiertas y los resultados cuantitativos.

Por otro lado, las recomendaciones para una docencia de emergencia fueron muy valiosas y dignas de ser consideradas en posibles estudios, así como el uso de las diferentes herramientas tecnológicas, ya que son muestra de lo que menciona Dussel (2020), es decir, la pedagogía de emergencia está usando varias plataformas digitales que son las que están más al alcance del docente y del estudiante. Sin embargo, es necesario analizar sus efectos sociotecnológicos en la configuración de las prácticas docentes.

\author{
Durante la docencia de \\ emergencia, la parte humana \\ y actitudinal es una necesidad \\ a la hora de afrontar el reto que \\ están viviendo tanto estudiantes \\ como docentes, por ello, se \\ recomienda la flexibilidad y la \\ empatía con el alumnado
}

Es importante mencionar que, durante la docencia de emergencia, la parte humana y actitudinal es una necesidad a la hora de afrontar el reto que están viviendo tanto estudiantes como docentes, por ello, se recomienda la flexibilidad y la empatía con el alumnado. A partir de los comentarios de los docentes se puede incluir lo planteado por Díaz Hoyos et al. (2020), quienes proponen modelos de capacitación en los que se integren tres elementos competenciales, como son competencias virtuales, pedagógico-didácticas y socioemocionalcomunicacionales.

\subsection{La relación que existe entre la capacitación del docente y su actitud tecnopedagógica}

Se pudo constatar la importancia de una actitud de autoformación y capacitación por parte del docente de manera continua. A su vez, busca estrategias para que puedan es- 
tablecerse mecanismos de enseñar a aprender y potenciar el autoaprendizaje; al igual que perfeccionar los cursos de capacitación mediante la reflexión y la mejora continua (Suárez, 2020). Los resultados de la categoría de actitudes contrastadas entre las dos encuestas muestran avances respecto a las posturas de integración de la tecnología, como elemento esencial en la docencia durante la pandemia. El ítem de mayor avance es el que se refiere a la valoración de las TIC como un recurso que apoya el aprendizaje, mientras que los menos valorados son que las TIC implican mayor tiempo y que son una distracción para el alumnado.

Por todo esto se puede concluir que, a partir de la capacitación, hubo un aumento proporcional de las actitudes respecto a la medición inicial. Esto se puede explicar debido a que el hecho de enfrentar al docente a experiencias de formación virtual ha permitido la adquisición de nuevas competencias y comprender mejor los roles que se esperan de un profesor virtual (Gros y Silva, 2005).

Díaz Hoyos et al. (2020) mencionan que muchos docentes, antes del 2020, manifestaban actitudes reticentes para el desarrollo de competencias digitales; principalmente, conocerlas, incorporarlas y aplicarlas a su práctica docente. Sin embargo, tras verse forzados por la pandemia, su actitud frente al uso de la tecnología y el desarrollo de sus competencias digitales ha cambiado.

Por lo tanto, las actitudes de los profesores juegan un rol importante a la hora de ver los resultados en el aula (Padilla, 2018). La actitud positiva y las competencias docentes son cruciales en estos momentos, por lo que hay una relación directa entre una actitud favorable y la formación en TIC.

\section{Conclusiones y futuras investigaciones}

En este estudio de investigación se han podido analizar las características de un curso de capacitación docente y se han detectado las necesidades de formación. Los resultados de este trabajo de investigación desvelaron una necesidad de actualización en saberes tecnopedagógicos por parte del personal académico del bachillerato mexicano. Se constató que, a inicios de la pan-
Los resultados de este estudio desvelaron una necesidad de actualización en saberes tecnopedagógicos por parte del personal académico del bachillerato mexicano demia, existía una necesidad imperante de capacitación. Se demostró que hubo avance en el desarrollo de competencias a partir del curso ofertado. Sin embargo, es cierto que los procesos de formación no se limitan a un curso virtual, sino que requieren de una oferta articulada y sistematizada que garantice la continuidad del desarrollo de competencias acordes a los intereses y a las necesidades del profesorado. 
Se identificó que existe una relación entre la capacitación del profesorado y la actitud positiva para incorporar las TIC en el aula. Esto se convierte en un factor en la habilitación de competencias digitales docentes.

Por otra parte, la presente investigación deja abierta algunas posibilidades y nuevas líneas de trabajo, como, por ejemplo, la búsqueda constante de mejoras en los cursos de capacitación docente.

Es importante detectar las necesidades de capacitación docente de acuerdo con el contexto que vive el académico. El docente, en la educación remota a distancia y virtual, se ha enfrentado a diversas problemáticas y necesidades que valdría la pena indagar.

Por último, como futuras líneas de investigación se plantea evaluar el impacto de esta modalidad emergente en el surgimiento de nuevos modelos y estrategias de enseñanza, así como las modificaciones de prácticas educativas en las instituciones.

Es importante indagar hasta qué punto el docente se ha visto forzado a aprender algunas competencias digitales y la reflexión del éxito de algunas acciones, que probablemente lo lleve a una innovación continua en el aula. Por lo tanto, se abren retos para la investigación educativa que pueden apoyar el surgimiento de posibles nuevos paradigmas de enseñanza-aprendizaje.

\section{Referencias bibliográficas}

Cabero Almenara, J., Marín Díaz, V. y Castaño Garrido, C. (2015). Validación de la aplicación del modelo TPACK para la formación del profesorado en TIC. @tic. Revista d'Innovació Educativa, 14, 13-22. https:// doi.org/10.7203/attic.14.4001

Cabrales, A., Graham, A., Sahlberg, P., Hodges, C., Moore, S., Lockee, B., Trust, T., Bond, A., Lederman, D., Greene, J., Maggioncalda, J., Soares, L., Veletsianos G. y Zimmerman, J. (2020). Enseñanza de emergencia a distancia: textos para la discusión. The Learning Factor. http://www.educac cionperu.org/wpcontent/uploads/2020/04/
Ensen\%CC\%83an-Remota-de-Emergen cia-Textos-para-la-discusio\%CC\%81n.pdf

Cardona-Londoño, C. M. ${ }^{a}$, Ramírez-Sánchez, M. ${ }^{a}$ y Rivas-Trujillo, E. (2020). Educación superior en un mundo virtual forzado por la pandemia del COVID-19. Revista ESPACIOS, 41(35), 44-57. http://www.revistaes pacios.com/a20v41n35/a20v41n35p04.pdf

Delgado, P. (2020). La capacitación docente, el gran reto de la educación en línea. Observatorio de Innovación Educativa. Tecnológico de Monterrey. https://observatorio.tec.mx/edunews/capacitacion-docente-covid 
Díaz Barriga, Á. (14 de mayo de 2020). Educación y pandemia: tensiones, retos y experiencias [vídeo]. Instituto de Investigaciones sobre la Universidad y la Educación/Universidad Nacional Autónoma de México. https://www. youtube.com/watch?v=tY6s54jE3il\&ab_ channel=IISUEUNAMoficial

Díaz Hoyos, J. A., Sánchez Sánchez, M.ª J., Aguilera Rodríguez, M. E., Loyola Polo, K. E., Ramírez Castro, J. A. y Reynosa Navarro, E. (2020). Capacitación docente y calidad educativa en tiempos de COVID-19. Revista Científica, Cultura, Comunicación y Desarrollo, 5(3), 84-89. https://rccd.ucf.edu. cu/index.php/aes/article/view/263

Dussel, I. (2020). La formación docente y los desafíos de la pandemia. Educación, Formación e Investigación, 6(10), 11-25. http://dgescba.edu.ar/wp/wp-content/ploads/2020/ 08/Dussel.pdf

Fardoun, H., González-González, C. S., Collazos, C. A. y Yousef, M. (2020). Estudio exploratorio en Iberoamérica sobre procesos de enseñanza-aprendizaje y propuesta de evaluación en tiempos de pandemia. Education in the Knowledge Society, 21(17). https:// doi.org/10.14201/eks.23537

Flores Peña, M. R. y Navarrete Cueto, C. A. (2020). Diagnóstico de necesidades de capacitación en el uso de plataformas virtuales ante la contingencia del COVID-19 en los estudiantes y docentes de Educación Media Superior Tecnológica. Dilemas Contemporáneos: Educación, Política y Valores, 8(5). http:// www.scielo.org.mx/scielo.php?pid=S2007$78902020000800017 \&$ script=sci_arttext

Gallardo, K. (21 de julio de 2020). Evaluación del aprendizaje en tiempos del COVID-19 [vídeo]. Observatorio de Innovación Educativa. https://www.youtube.com/watch?v=Mf xE6CjJIE0\&ab_channel=Observatoriodelnno vaciónEducativa

Gros Salvat, B. y Silva Quiroz, J. (2005). La formación del profesorado como docentes en los espacios virtuales de aprendizaje. Revista Iberoamericana de Educación, 36(1), 1-13.

Guayara Cuéllar, C. T., Millán Rojas, E. E. y Gómez Cano, C. A. (2019). Diseño de un curso virtual de alfabetización digital para docentes de la Universidad de la Amazonia. Revista Científica, 1(34), 34-48.

Hodges, C., Moore, S., Lockee, B., Trust, T. y Bond, A. (2020). The difference between emergency remote teaching and online learning. Educause Review, 27, 1-12. https:// medicine.hofstra.edu/pdf/faculty/facdev/fac dev-article.pdf

INEE. (23 de julio de 2018). La educación media superior en México [vídeo]. https://www.inn ee.edu.mx/la-educacion-media-supe rior-en-mexico/

Koehler, M. J. y Mishra, P. (2008). Introducing TPCK. Handbook of Technological Pedagogical Content Knowledge (TPCK) for Educators. AACTE Committee on Innovation and Technology (Ed.) (pp. 3-29). Routledge; Taylor \& Francis Group for the American Association of Colleges of Teacher Education. https://www.punyamishra.com/wpcontent/ uploads/2008/05/koehler_mishra_08.pdf

Lima Villeda, D. N. y Flores Macías, R. C. (2018). Conocimientos del tutor en línea en una universidad pública mexicana: Modelo TPACK. Hamut'ay, 5(2), 22-33. https://doi. org/10.21503/hamu.v5i2.1618

Lozano Medina, A. (2016). La formación docente en la educación media superior en México. Retos y posibilidades. XI Seminario Internacional de la Red Estrado. http://redeestrado. org/xi_seminario/pdfs/eixo3/99.pdf

Luca, M. P. de. (2020). Las aulas virtuales en la formación docente como estrategia de continuidad pedagógica en tiempos de pandemia. Usos y paradojas. Análisis Carolina, 33, $1-12$. 
Medrano, V., Eduardo, Á. y Castillo, Y. (2015). Capítulo 3. Formación de los docentes de educación básica y media superior. En A. Santos del Real y A. Delgado Santoveña (Eds.), Los docentes en México. Informe 2015 (pp. 83-123). Instituto Nacional para la Evaluación de la Educación. https://historico. mejoredu.gob.mx/wp-content/uploads/ 2018/12/P1|240.pdf

Mishra, P. y Koehler, M. J. (2006). Technological pedagogical content knowledge: A new framework for teacher knowledge. Teachers College Record, 108(6), 1.017-1.054. https://www. punyamishra.com/wp-con tent/uploads/2008/01/mishra-koehler-tcr 2006.pdf

Morán Peña, F. L., Morán Peña, F. E. y Albán Sánchez, J. D. (2017). Formación del docente y su adaptación al modelo TPACK. Revista Ciencias Pedagógicas e Innovación, 5(1). https://doi.org/10.26423/rcpi.v5i1.154

Mohamad Nasri, N., Husnin, H., Mahmud, S. N. D. y Halim, L. (2020). Mitigating the COVID19 pandemic: a snapshot from Malaysia into the coping strategies for pre-service teachers' education. Journal of Education for Teaching, 1-8. https://doi.org/10.1080/026 07476.2020.1802582

Padilla Partida, S. (2018). Usos y actitudes de los formadores de docentes ante las TIC. Entre lo recomendable y la realidad de las aulas. Apertura, 10(1), 132-148. http:// dx.doi.org/10.32870/Ap.v10n1.1107
Peña, R. F., Waldman, F. B., Soneyra de Pérez Berbain, N. M., Tejada, G. A., Carrere Cadirant, G. S., Passaglia, J. y Contrera, M. (2012). Implementación de los entornos virtuales de aprendizaje en cursos de capacitación docente. Revista Iberoamericana de Educación, 60(1), 117-128.

Picón, G. A., Karina, G., Caballero, G. de y Paredes, N. (2020). Desempeño y formación docente en competencias digitales en clases no presenciales durante la pandemia COVID-19. Human Sciences. 1-16. https://doi.org/10. 1590/SciELOPreprints.778

Sánchez Mendiola, M., Martínez Hernández, A. M. ${ }^{a}$, Torres Carrasco., R., Agüero Servín, M. ${ }^{a}$ M. de, Hernández Romo, A. K., Benavides Lara, M. A., Jaimes Vergara, C. A. y Rendón Cazales, V. J. (2020). Retos educativos durante la pandemia de COVID-19: una encuesta a profesores de la UNAM. Revista Digital Universitaria (RDU), 21(3), 1-24. http:// doi.org/10.22201/codeic.16076079e.2020. v21n3.a12

Shulman, L. (1987). Knowledge and teaching: foundations of the new reform. Harvard Educational Review, 57(1), 1-23.

Suárez, N. (2020). Formación docente universitaria y crisis sanitaria COVID-19. CienciAmérica, 9(2), 109-114.

UNESCO-UNICEF. (2020). What Have We Learnt? Overview of Findings from a Survey of Ministries of Education on National Responses to COVID-19. https://openknowledge. worldbank.org/handle/10986/34700 